\title{
High Temperature NDIR Gas Measurement Module
}

\author{
Eick, Thomas; Magi, André; Sausemuth, Olaf; Biermann, Steffen; Sachse, Patrick \\ Micro-Hybrid Electronic $\mathrm{GmbH}$ \\ Heinrich-Hertz-Straße 8, 07629 Hermsdorf
}

\section{Introduction}

Gas measurement modules are used in several fields of science and technology for example in the chemical or biochemical industry, in the environmental measurement technique or in the check of work places in relation to the concentration of unhealthy gases. Measuring modules for example for the determination of $\mathrm{CO}_{2}$ contents in air are meanwhile offered by several providers. Normally the modules are working at moderate temperatures for example up to $60^{\circ} \mathrm{C}$. However, scientists and engineers are more and more interested in high temperature gas measuring modules.

For these applications Micro-Hybrid Electronic $\mathrm{GmbH}$ Hermsdorf has developed a gas measuring module that is able to withstand temperatures up to $190^{\circ} \mathrm{C}$. The module is based on the well known NDIR (nondispersive infrared) principle.

\section{Principle of NDIR Gas Measurement}

Figure 1 shows the principle of the NDIR measurement method.

The measuring chamber is filled with the gas to be investigated. Infrared radiation delivered by a source is passing the gas and is generating a signal voltage in the thermopile infrared detector. The detector is carrying a narrow band filter having its transmission at the same wavelength that is absorbed by the gas to be detected. In this way the presence of the investigated gas inside of the chamber causes a drop of the output signal of the detector.

\section{Prototype of the Gas Measuring Module}

\subsection{Measuring Chamber}

Figure 2 shows a three dimensional model of the gas measuring chamber that was developed by MicroHybrid. In the actual configuration the module is made for measuring $\mathrm{CO}_{2}$.

On one side the source is located and on the other side the detector. A dual channel thermopile infrared detector is used with two narrow band infrared filters. The first filter has a transmission at $4.26 \mu \mathrm{m}$ the main absorption wavelength of $\mathrm{CO}_{2}$. The other filter has its transmission at $4,00 \mu \mathrm{m}$ a wavelength that is not influenced by any usual gases. It is used as a reference channel to correct possible changes of the intensity of the source that can occur for example by aging effects or by the influence of dust and smoke. Holes in the side wall enable the gas to get into the chamber.

Figure 3 shows the absorption lines of $\mathrm{CO}_{2}$ in the wavelength range near $4.26 \mu \mathrm{m}$ together with the transmission curve of the used infrared filter.

\section{$\underline{3.2 \text { Infrared Source }}$}

A special infrared source was developed for working at high ambient temperatures. The active element of the source is a spiral that is made from a thin metal sheed with the help of laser means. The effective area is $3.8 \mathrm{~mm}^{2}$. The spiral is mounted inside of a TO39 housing by welding it onto the pin ends of the socket inside of the housing. An open cap is used without infrared filter. The infrared source is able to withstand the required high temperatures. Figure 4 shows the used spiral-wound filament radiator. The TO39 housing is carrying a reflector made from aluminium to increase the given infrared power. The radiator is working with an electric power of about $1 \mathrm{~W}$ and therefore gives a very powerful infrared radiation. 


\section{$\underline{3.3 \text { Infrared Detector }}$}

The used dual thermopile detector contains two thermopile chips one for the $\mathrm{CO}_{2}$ and one for the reference channel (see figure 5). This detector is especially made to withstand high ambient temperatures up to $190^{\circ} \mathrm{C}$. The thermopiles inside are produced in a special technology that makes them resistant against high temperatures.

The infrared filters at the front of the detector are metallized and soldered inside of the cap by a special technology.

\section{Measuring method}

The measuring chamber that is situated in the hot environment is connected with the electronic circuit at room temperature by a high temperature resistant cable that contains twisted and shielded pairs of wires. The cable is used on the one hand for the power supply of the infrared source and on the other hand for the connection of the thermoelectric signal voltage of the detectors with the amplifier and the evaluation electronic. To elliminate disturbing voltages in the signal the infrared source is switched on and off periodically and only the difference of the signal voltage between both states is registered. A special electronic evaluation method is used to elliminate disturbing voltages from external fields or noise voltages.

The absorption of the $\mathrm{CO}_{2}$ at a given concentration depends on the temperature because of the temperature dependence of the $\mathrm{CO}_{2}$ density. Figure 6 shows the concentration dependence of the

absorption of the $\mathrm{CO}_{2} \quad A=1-\frac{U_{\mathrm{CO} 2}}{U_{\text {ref }}} \quad$ measured at a temperature of $25^{\circ} \mathrm{C}$.

The sensitivity of the detector is decreasing with increasing ambient temperature because of the temperature dependence of the Seebeck coefficient of the thin film thermocouples. Figure 7 shows the measured normalized signal voltage of the $\mathrm{CO}_{2}$ channel versus temperature.

Furthermore the filter transmission curves show a slight shift with respect to the wavelength in dependence on temperature.

That's why the module must be calibrated for the special temperature range where it shall be used for application.

\section{$\underline{5 .}$ Test results}

The module was calibrated with the help of test gases of a defined concentration. The measurement accuracy was found to be $2 \%$ of the nominal value of the range. 


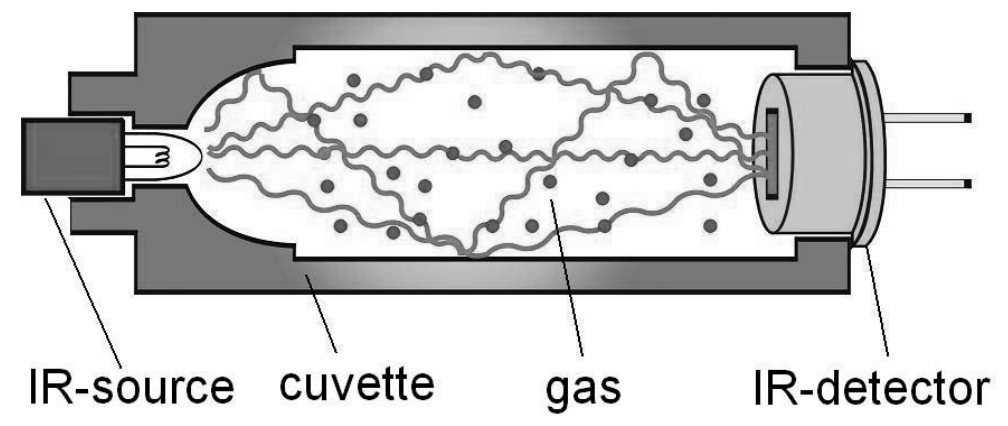

Figure 1: $\quad$ Principle of the NDIR measurement method.

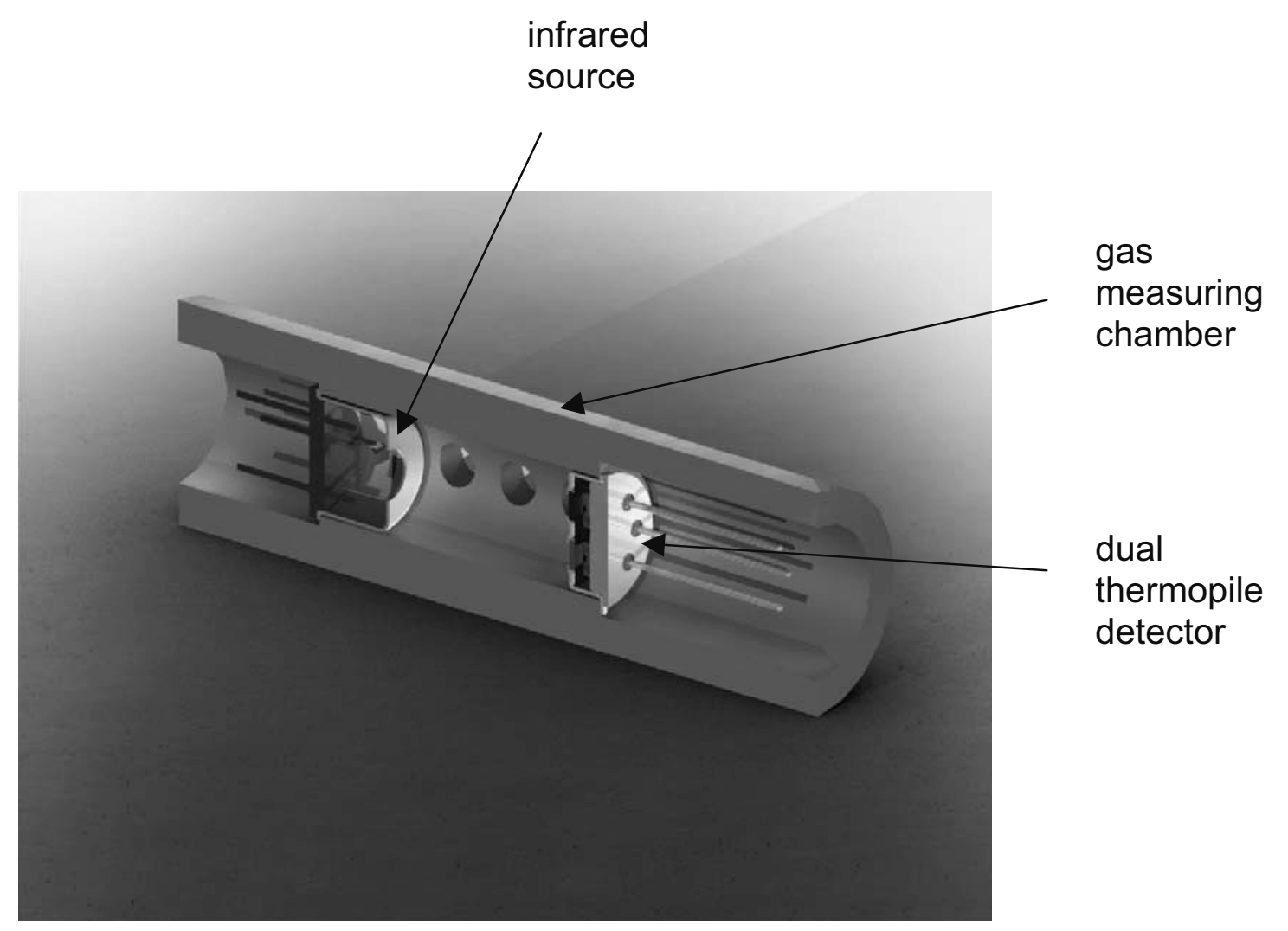

Figure 2: $\quad$ Three dimensional model of the prototype of the gas measuring chamber. 


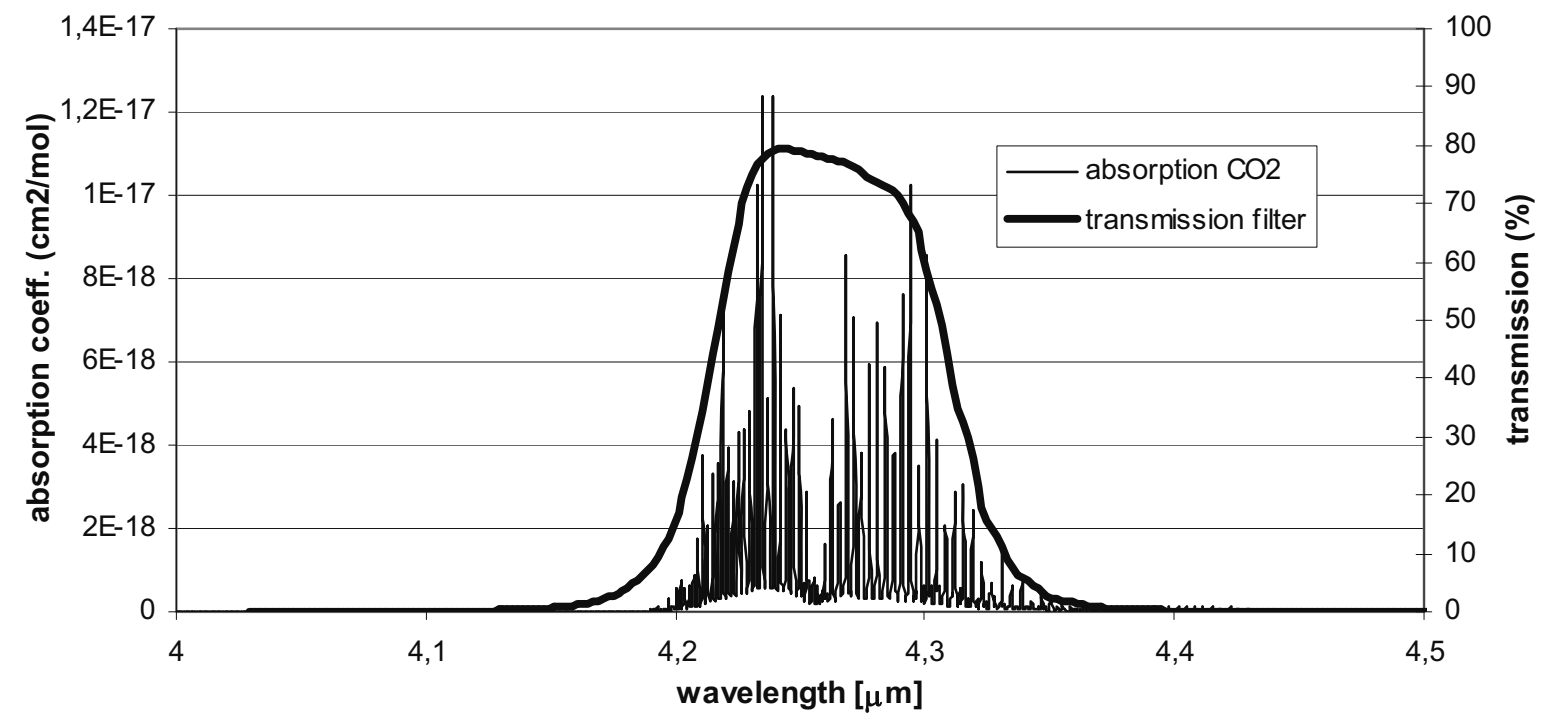

Figure 3: Absorption of $\mathrm{CO}_{2}$ in dependence on the wavelength and transmission curve of the used filter.

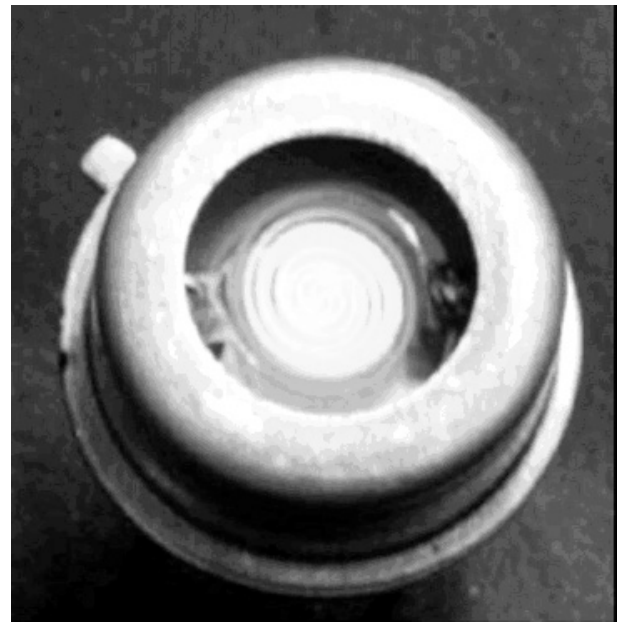

Figure 4: Spiral-wound filament radiator. 


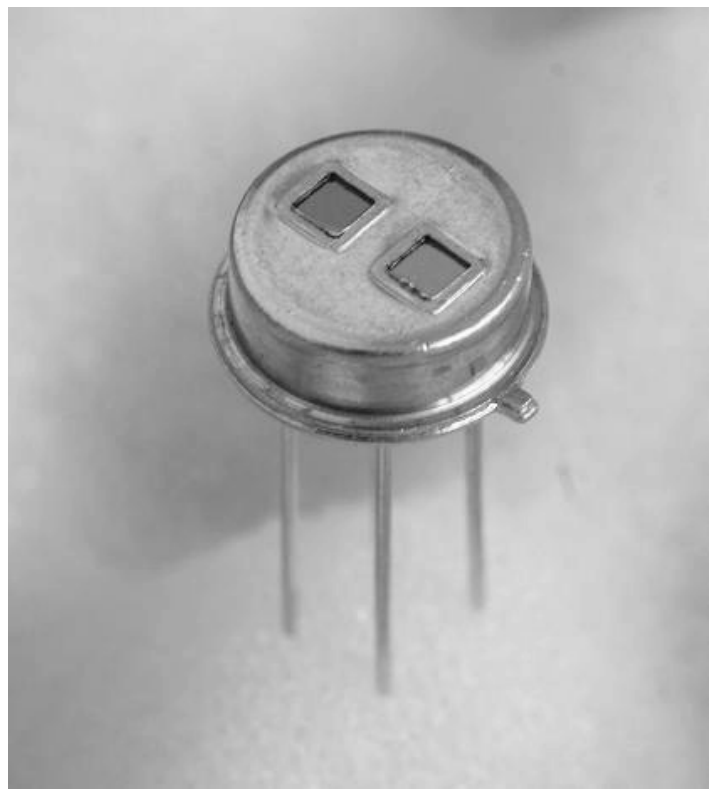

Figure 5: Dual detector with $\mathrm{CO}_{2}$ and reference filter.

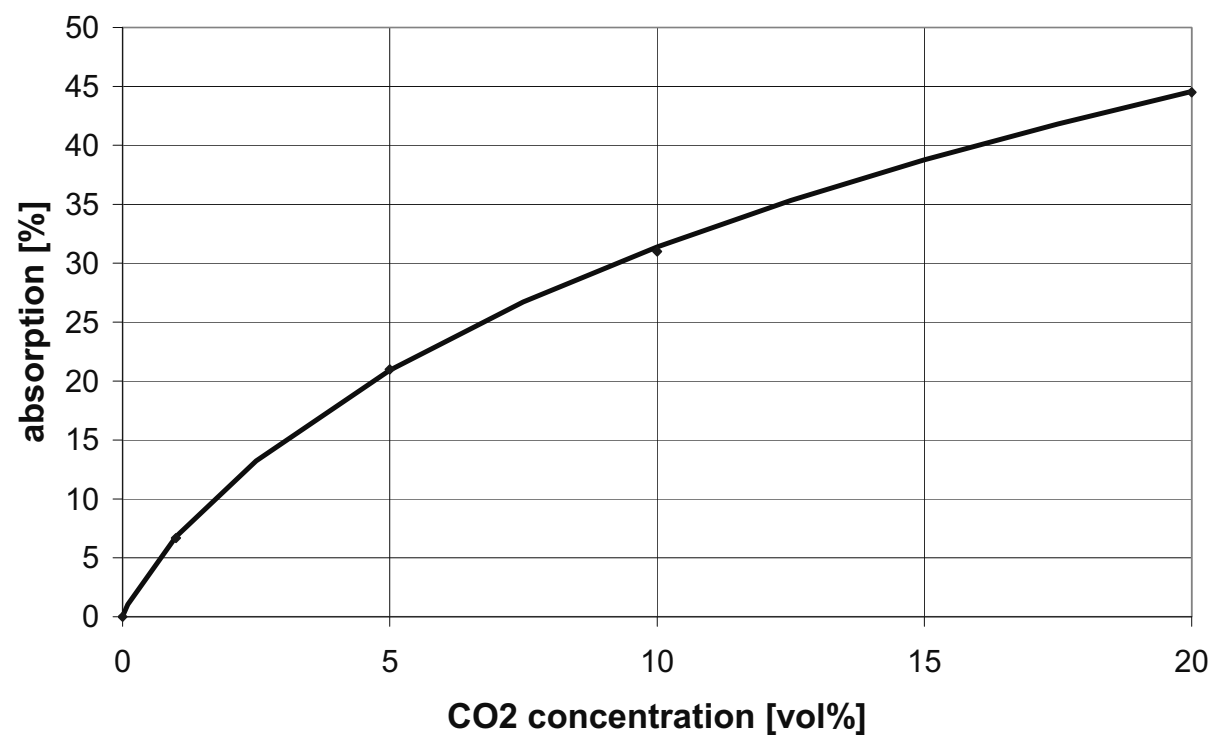

Figure 6: $\quad$ Measured concentration dependence of the absorption of the $\mathrm{CO} 2$ gas inside of the chamber. 


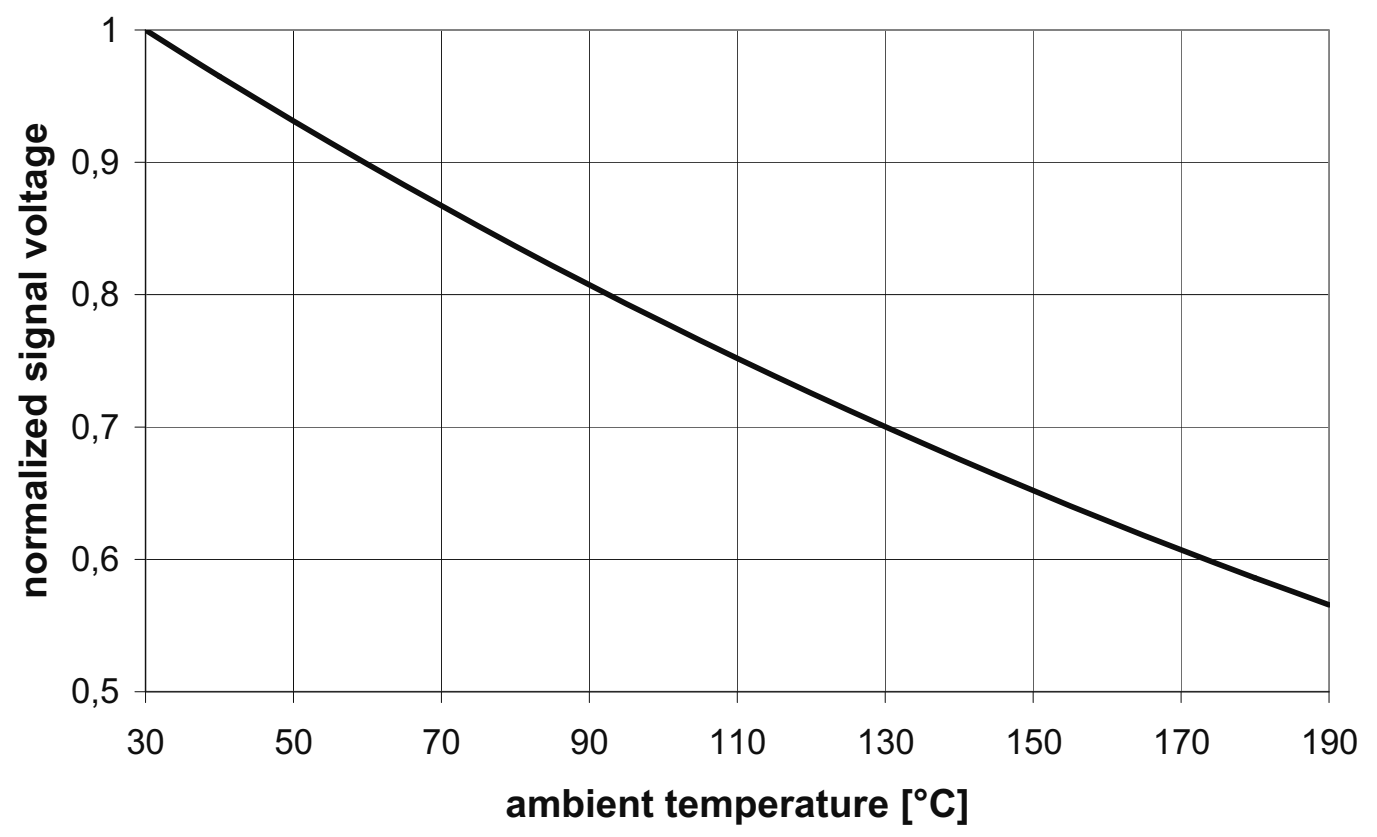

Figure 7: Temperature dependence of the normalized signal voltage of the $\mathrm{CO}_{2}$ channel. 